\title{
Vitamin A Rich Foods Consumed For Dietary Intake and Supplementation Among Children Aged 12 To 59 Months in Gatunga Ward, Tharaka Nithi County, Kenya
}

\author{
Nkingo Christine Kananu*, Judith Waudo*, Eunice Njogu* \\ Department of Food, Nutrition and Dietetics, Kenyatta University, Nairobi, Kenya \\ Email address: \\ cknkingo3@gmail.com (N. C. Kananu),waudo.judith@ku.ac.ke(J. Waudo),njogu.eunice@gmail.com (E. Njogu) \\ ${ }^{*}$ Corresponding author
}

To cite this article:

Nkingo Christine Kananu, Judith Waudo, Eunice Njogu. Vitamin A Rich Foods Consumed For Dietary Intake and Supplementation Among Children Aged 12 To 59 Months in Gatunga Ward, Tharaka Nithi County, Kenya. Central African Journal of Public Health.

Vol. 6, No. 2, 2020, pp. 71-79. doi: 10.11648/j.cajph.20200602.14

Received: November 15, 2019; Accepted: December 6, 2019; Published: February 21, 2020

\begin{abstract}
Vitamin A food sources intake is one of the dietary interventions that support the achievement of nutrition priority outcomes. Inadequate Vitamin A intake leads to its deficiency which causes depressed immune response, impaired movement of iron, poor growth, night blindness and xerophthalmia which are a major public health concerns. The study objective was to establish Vitamin A rich foods consumed by children aged 12 to 59 months. The target population was 370 caregivers of children aged 12 to 59 months who were clustered and sampled using purposive cluster and random sampling. A cross sectional descriptive study design was used to guide this study. Primary data was collected using semi-structured questionnaires and key informant interviews schedules from caregivers and key informants respectively. Quantitative data was analysed using Statistical Package for Social Sciences Version 20 while qualitative data was organized and analysed thematically. The findings revealed that only $41.8 \%$ consumed more than five food groups and indicator of a children likelihood of suffering from Vitamin A deficiency due to limited dietary diversity. It also emerged that only 36 (9\%) of the respondents could mention at least one type of foods rich in Vitamin A while only (23.5\%) could mention at least one benefit of Vitamin A, an indicator of knowledge on Vitamin A-rich foods and their benefits was scanty. The study recommends that the government and stakeholders need to develop a policy of ensuring caregivers are adequately educated on Vitamin A-rich foods and their benefits. Interventions such as forming self-help groups which will provide platforms of starting of income generating activities such as investing in production of green vegetables, fruits and chicken to diversify on Vitamin A rich Vitamin A-rich foods.
\end{abstract}

Keywords: Vitamin A-rich Foods Sources, Food Groups, Dietary Diversity, Consumption of Vitamin A foods, Cross Sectional Descriptive Study Design

\section{Introduction}

Vitamin $A$ is essential in enhancing eye vision, reproduction, cell division, and differentiation [1]. According to the United Nations Children's Fund (UNICEF), Vitamin A is important for a strong immune system and healthy growth and development of children [2]. Further, if there is inadequate intake of Vitamin $\mathrm{A}$ in children, there is an increased risk to illness, blindness, death, measles, and diarrhoea. Vitamin A maintains the surface linings of the eye, respiratory, urinary, and intestinal tracts and is essential for the functioning of an individual's immune system [3]. According to the World Health Organisation (WHO), Vitamin A is essential for proper foetal development right from the embryonic stage [4]. From these observations optimal Vitamin A status is important for maintenance of good health and prevention of disease.

VAD symptoms may include loss of appetite, hair loss, rashes and frequent infections especially respiratory related [3]. Mostly, VAD symptoms may also present as dry skin and eyes, night blindness and other visual challenges, fatigue, poor growth and development among others [5]. Primarily, 
Vitamin A deficiency is caused by prolonged dietary deprivation and is common in areas where main consumed staple foods have insufficient levels of carotene. Another cause of VAD is inadequate metabolic conversion of carotene to Vitamin A in the body which is as result of poor absorption, storage, and transport of Vitamin A.

Children aged between 5 and 59 months and pregnant women are two major groups who are at risk of suffering from VAD. Children aged between 5 and 59 months and pregnant women are likely to suffer from VAD since they fall in the groups in the society affected by major nutritional concern in poor societies, especially in lower income countries [6]. The young children and pregnant women are vulnerable to Vitamin A-rich dietary decline which predisposes VAD and its disorders. VAD manifest in a person when insufficient Vitamin A is consumed in a diet as too little is absorbed by the body from the food sources, or too much is lost due to illness or infection [7,8]. Motee and Jeewon observe that for young children, breast milk is a good source of Vitamin A, however, lack of exclusive breastfeeding and abrupt weaning may contribute to VAD [9]. According to Hazir et al. wrong timing of introduction of complementary feeding deprives an infant optimum nutrition and this can lead to morbidity, increased mortality and under nutrition [10]. Timeliness is evaluated by determining whether infants are getting solid, semi-solid, and soft foods in addition to being breastfed.

According to Chiu and Watson (2015) poor Vitamin A source diets and disease infections coexist and interact in populations where VAD is rampant [11]. They (Chiu \& Watson) explained that in such situations, Vitamin A deficiency has a high likelihood of increasing infection severity. They also noted that infection severity in turn, can reduce intake and accelerate body losses of Vitamin A subsequently leading to VAD deficiency in the body. According to Akhtar et al. VAD occurs commonly in developing countries in an environment of ecological, social, and economic deprivation [8]. In these environments deficient dietary intake of Vitamin A is associated with severe infections such as measles, diarrhoea and respiratory diseases. Sardesai attributed depressed appetite low absorption, excessive metabolism and excretion to low intake of Vitamin A [12]. Vitamin A deficiency has been noted to be a major contributor of under-five mortality and provision of adequate Vitamin A supplementation could prevent many deaths [13].

There are a number of interventions adopted to address VAD. Nair, Augustine and Konapur posit that the primary intervention measure is to avail adequate Vitamin A intake through dietary diversification [14]. This primary intervention measure is more feasible if properly promoted in the communities with high prevalence of severe VAD. The Vitamin A diet sources comprise of animal and non-animal (plant based) sources. Plant based Vitamin A sources as opposed to animal based ones constitutes the largest proportion of foods intake for most individuals in the developing world. However, these plant based food sources do not provide enough active Vitamin A. Jemberu, Zegeye, Singh and Abebe argue that due to insufficient Vitamin A intake from plant food sources, the only feasible remedy is to provide dietary diversification [15]. This would require increased consumption of animal-based Vitamin A food sources (bioavailable Vitamin A-rich foods) together with nutritious fruits and vegetables.

WHO estimates, over 250 million children under five years of age globally suffer from VAD which as a result has caused blindness to $250-500$ millions of them [16]. Kenya is one of the Sub-Sahan country that is affected by VAD. A report by Micronutrient Survey of 2011 in Kenya, 76\% of the children below five years were found to suffer from Vitamin A deficiency [17]. A Kenya Ministry of Public Health and Sanitation (MoPHS) survey estimated the prevalence of VAD to be $84 \%$ among children below five years [18].

The health survey established that $64.4 \%$ of children aged $6-23$ months in Eastern Province consumed foods rich in VA [18]. These findings were captured for the entire Eastern Province but did not specifically indicate figure for Tharaka Nithi County. This is could be not a true reflection of the situation on the ground bearing in mind the difference in socio-economic disparities. The Vitamin A supplementation coverage in Sub-Saharan countries is major concern for governments and stakeholders.

\section{Method}

\subsection{Ethical Considerations}

Research approval, ethical clearance and research permit were granted by Kenyatta University Graduate School, Kenyatta University Ethical Review Committee and National Council of Science, Technology and Innovation respectively. Informed consent was obtained from respondents. Finally permission to collect data was granted by the National and County government.

\subsection{Study Design}

The study adopted a cross sectional descriptive study design. This research design was appropriate for determining association between the independent and dependent variables of the study. The study was carried out with the use of a semi structured questionnaires to determine the VA intake, adequacy of food taken and the VA supplement taken by the children aged between 12 to 59 months.

\subsection{Study Participants}

The research involved the caregivers of the children aged 12 to 59 months who had resided in Gatunga Ward for at least 12 months. However, mothers or caregivers whose children have lived in the area of study for less than 6 months were not included in the study.

\subsection{Study Implementation}

Eight research assistants with a minimum qualification of 
secondary school level of education certificate were recruited from the study area and trained by the principal researcher on the purpose and objectives of the study. They were trained on the study objectives, methodology, interview skills and maintenance ethics during and after data collection. They were also trained on administering data collection instrument, interviewing techniques, actual data collection and recording, ethics and. Respondents (caregivers) filled questionnaires with the help of research assistants. As well, key informants were orally interviewed by the researcher using interview guide.

\subsection{Statistical Analysis}

Primary data collected using questionnaires was cleaned, coded and then keyed into Statistical Package for Social Sciences (SPSS) Version 20 computer software used for data analysis. Mean, variance, standard deviation and percentages descriptive statistics were computed to analyse quantitative primary data collected using questionnaires. Analysed quantitative data was organized and summarized using tables and charts. Vitamin A coverage was determined by dividing the number of children receiving VAS in the past 12 months by the total number of children aged 12 to 59 months in the study area then multiplied by 100 . The influence of independent variables on the intake of Vitamin A was tested using Chi square where a $\mathrm{p}$ value of $<0.05$ was considered significant. A Chi-Square $p$ value of more that is 0.05 was an indicator that tested variables did not vary meaning there was no statistical difference among the group variables. Chi Square $p$ values equal or less than 0.05 indicated the tested variables varied significantly - meaning there was statistical difference among the group variables explored.

\section{Results}

The study objective sought to determine Vitamin A-rich foods consumed by children aged 12 to 59 months in Gatunga Ward. Vitamin A is sourced from plants and animal products whose availability is determined by socio-economic factors. The study findings on the dietary intake for Vitamin A are presented in sections below.

\subsection{Vitamin A-Rich Foods Identified by the Caregivers of Children Aged 12-59 Months}

The respondents were asked to indicate the kind of Vitamin A-rich foods they feed their children aged 12 to 59 months. This was necessary in order to establish respondents' knowledge of Vitamin A-rich foods consumed by children aged 12 to 59 months (Table 1).

Table 1. Vitamin A-rich foods identified by the caregiver.

\begin{tabular}{lll}
\hline \multirow{2}{*}{ Vitamin A-rich foods } & Responses & Percentage within cases \\
\cline { 2 - 3 } & Count (n) & 78.9 \\
\hline Eggs & 30 & 60.5 \\
Meat & 23 & 31.6 \\
Milk & 12 & 23.7 \\
Fruits (Mangoes and Pawpaw) & 9 & 10.5 \\
Fish & 4 & 205.3 \\
Total & 78 & \\
\hline
\end{tabular}

NB: Data was analysed from multiple responses.

The analysed data in Table 1 found that most 30 (38.5\%) identified eggs as one type of food rich in Vitamin A as opposed to $4(5.1 \%)$ who named fish. The findings imply that the respondents get Vitamin A mostly from animal sources. However, the responses who responded to question were few, an indicator that knowledge of the foods rich in vitamin was scanty. Of important to note, no single respondent identified any Vitamin A-rich foods from vegetable sources.

\subsection{Sources of Food Rich in Vitamin A Consumed by Children in Households}

The respondents were asked to indicate the sources of food they thought was rich in Vitamin A. This was necessary in order to establish if the respondents produce them in their farms or not (Table 2).

Table 2. Sources of Vitamin A-rich foods in the households.

\begin{tabular}{lll}
\hline \multirow{2}{*}{ Sources of Food rich in Vitamin A } & Responses & Percent of Cases \\
\cline { 2 - 3 } & N & 92.9 \\
\hline Own reared chicken for meat and eggs & 289 & 76.8 \\
Own cows/goats milk & 239 & 71.4 \\
Buy vegetables from market and neighbours & 222 & 24.8 \\
Grow vegetables in my farm & 77 & 17.7 \\
Buy fruits from market and neighbours & 55 & 11.3 \\
Grow fruits in the farm & 35 & 10.3 \\
Buy meat/liver from butchery & 32 & 305.1 \\
Total & 949 & \\
\hline
\end{tabular}

The findings in Table 2 shows that most 289 (30.5\%) and $239(25.2 \%)$ of the respondents indicated that they rear chicken and keep cows or goats as source of Vitamin A respectively. The study also established that $222(23.4 \%)$ buy 
vegetables to get Vitamin A. The findings revealed that few $77(8.1 \%)$ and $35(3.7 \%)$ respondents grow crops rich in Vitamin A. Worth noting is a small number $32(3.4 \%)$ of the respondents who buy meats as source of Vitamin A. This can be attributed to the high cost of buying meats due to low income come levels as noted by this study.

The study through an oral interview with an Assistant Chief sought to establish the frequency of consumption of meat by residents of study area from their own reared chicken and meat bought from butcheries. The interview found that the residents rarely consume the animals they keep which are only slaughtered in special days and during festivities. However, consumption of eggs is common in households that keep chicken. The interview also noted that consumption of meat bought from butcheries is low and only persons with relatively good income can afford. The interviewee confided that most of the residents get Vitamin A-rich food from milk or from vegetables bought in the market.

\subsection{Vitamin A-rich Food Groups Consumed by Children Aged 12 to 59 Months}

The respondents were asked to identify Vitamin A consumed foods from the list. This was necessary to assess the intake of Vitamin A based food groups as recommended by FANTA (2009) [19]. Respondents results are presented in Table 3.

Table 3. Vitamin A-rich food groups consumed by children aged 12 to 59 months.

\begin{tabular}{lll}
\hline \multirow{2}{*}{ IDDS for Children Food Groups (Score: 0-8) } & Food Eaten/Taken \\
\cline { 2 - 3 } & Yes (\%) & NO (\%) \\
\hline Vitamin A-rich plant foods (Green leafy vegetables) & 58.5 & 41.5 \\
Eggs (all types of eggs) & 52.1 & 47.9 \\
Other fruits or vegetables (tomatoes, pumpkins) & 43.4 \\
Meat, poultry, fish, seafood (all types of meats) & 33.8 & 56.6 \\
Foods cooked in oil/fat (Fat, vegetable oils, margarine, ghee (traditional) & 32.5 & 66.2 \\
Milk and milk products (all types of milk products) & 25.7 & 67.5 \\
Roots or tubers (yellow sweet potatoes) & 22.5 \\
Fruits (Fruit mangoes, pawpaw, watermelon & 7.2 \\
\hline
\end{tabular}

From the eight food groupings assessed, the findings in Table 3 show that the children consumed a variety of Vitamin A-rich foods groups. Vitamin A-rich plant food (Green leafy vegetables) $58.5 \%$ were the most consumed Vitamin A-rich foods. On the other hand fruits (7.2\%) and roots or tubers $(25.7 \%)$ were among the least consumed Vitamin A-rich foods groups. From the findings above it is apparent that Vitamin A-rich plant foods (leafy vegetables) and eggs accounted for majority source of Vitamin A.

\subsection{Seven-Day Frequency of Vitamin A-Rich Foods Consumed by Children Aged 12-59 Months}

The respondents were asked to identify Vitamin A-rich foods from the list which were consumed children aged 1259 months in a period of one week ( 7 days). The study also sought how many times the children consumed various Vitamin A-rich foods. This was necessary in order to establish the frequency of consumption of Vitamin A-rich foods by children aged 12-59 months (Table 4).

Table 4. Frequency of Vitamin A-rich foods consumed in the previous seven days.

\begin{tabular}{|c|c|c|c|c|c|c|c|c|c|}
\hline Food taken & Once & Twice & Thrice & $\begin{array}{l}\text { Four } \\
\text { Times }\end{array}$ & $\begin{array}{l}\text { Five } \\
\text { Times }\end{array}$ & Six Times & $\begin{array}{l}\text { Seven } \\
\text { Times }\end{array}$ & $\begin{array}{l}\text { Total } \\
\text { consumption }\end{array}$ & Did not take \\
\hline Green leafy vegetables & 10.6 & 24.4 & 15.1 & 6.4 & - & - & 1.9 & 58.5 & 41.5 \\
\hline Carrots & 8.4 & 14.8 & 25.1 & 14.1 & 8.0 & 1.6 & 10.9 & 83.0 & 17.0 \\
\hline Fruits mangoes & 3.5 & 0.3 & - & - & - & - & - & 3.9 & 96.1 \\
\hline Pawpaw & 4.2 & 1.3 & 4.3 & - & - & - & - & 10.0 & 90.0 \\
\hline Watermelon & 1.9 & - & - & 3.2 & - & - & - & 5.1 & 94.9 \\
\hline Fats & - & 1.6 & - & 3.9 & 5.5 & 4.2 & 17.7 & 32.8 & 67.2 \\
\hline Vegetable oils & - & 1.0 & 3.2 & 3.9 & 1.3 & 6.8 & 47.9 & 64.0 & 36.0 \\
\hline Margarine & 26.7 & 13.8 & 7.4 & 1.0 & - & 1.0 & 2.3 & 52.1 & 47.9 \\
\hline Ghee (Traditional) & - & - & - & - & - & - & - & - & - \\
\hline Eggs & 26.7 & 13.8 & 7.4 & 1.0 & - & 1.0 & 2.3 & 52.1 & 47.9 \\
\hline Beef & 31.8 & 15.4 & 3.2 & - & - & - & 2.3 & 52.7 & 47.3 \\
\hline Chicken & 15.4 & 0.3 & - & - & - & - & - & 15.8 & 84.2 \\
\hline Pumpkin & 0.6 & 1.6 & 1.0 & 1.6 & 2.3 & 0.6 & 0.3 & 8.0 & 92.0 \\
\hline Sweet potatoes & 15.8 & 6.1 & 0.3 & 0.3 & - & - & 2.3 & 24.8 & 75.2 \\
\hline Milk & 2.9 & 18.0 & 3.5 & 2.3 & 1.3 & 1.6 & 7.4 & 37.0 & 63.0 \\
\hline
\end{tabular}

The findings above findings show mixed responses on the frequency of consumption of different rich foods rich in Vitamin A in a period of one week. It was established that cooking oil (64.0\%) were highest consumed Vitamin A-rich foods every day while pumpkin $(0.3 \%)$ was the least. The study also established that out of the 15 food rich in Vitamin A explored only ghee (traditional) was not consumed. 


\subsection{Dietary Diversity Assessment}

The dietary diversity assessment was done by computing dietary diversity scores using simple count of food groups that the children consumed over the preceding seven days using simple dietary diversity questionnaire. FANTA (2009) recommends an eight (8) food diversity food groups to assess
Vitamin A intake for children [19]. The adequacy of Vitamin A intake was determined by calculating Individual Dietary Diversity Score (IDDS) from the consumed foods collected using 24 hours recall questionnaires. The collected data was analysed for IDDS (Figure 1).

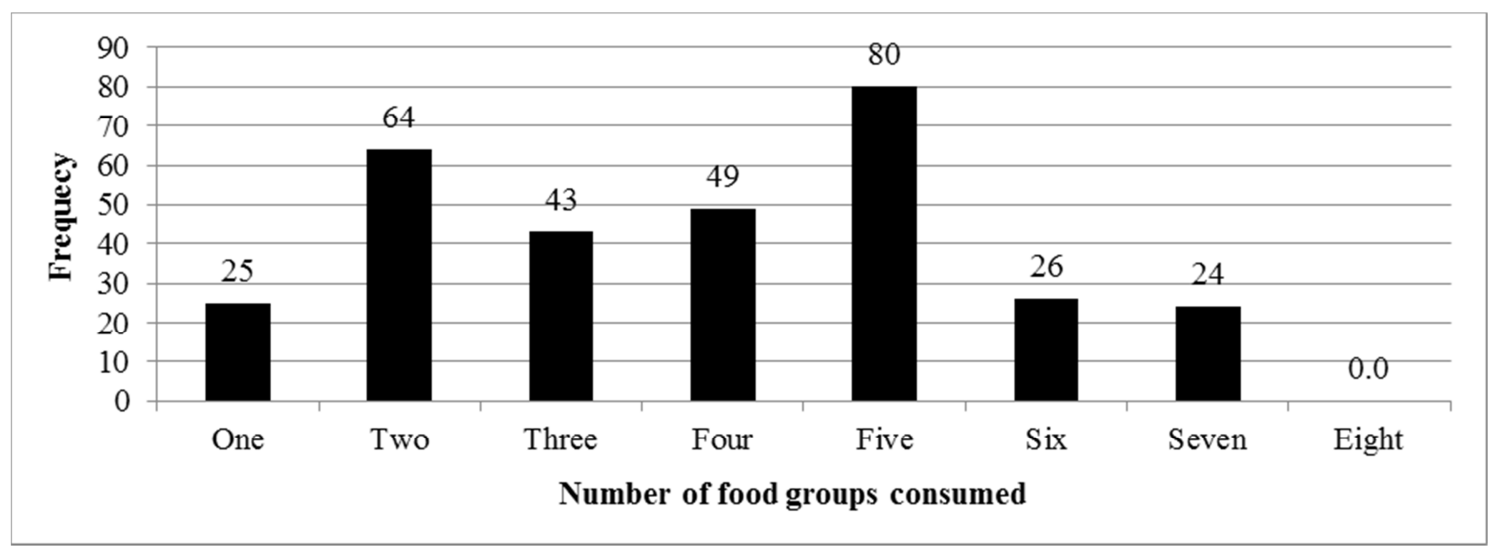

Mean 3.9, Standard deviation 1.72.

Figure 1. Individual dietary diversity score.

The findings above show that the most 80 of the children consumed five groups of foods followed by 64 who consumed two groups. The mean IDDS was (Mean 3.9, SD 1.72) from a possible eight food groups. The mean of 3.9 denote a scenario where the children are consuming low number of food groups. Only less than 130 consumed more than five and above food groups.

\section{Discussions}

From the study it emerged that the caregivers had little knowledge of the foods rich in Vitamin A which they feed the children. This was so because only a small proportion $(\mathrm{N}=78)$ responded to the questions posed. It also emerged that the respondents were not aware that there are plant based foods as source of Vitamin A. This was evidenced by the fact no caregiver identified such foods. The study also revealed that the caregivers only identified meat based food sources for Vitamin A. This was attributed to lack of adequate knowledge of the diversity of sources of Vitamin A. This was confirmed by one of the health promoters who indicated that caregivers have a notion that Vitamin A can only be found in meats. This observation explains why most of the respondents identified eggs and meats as the major foods rich in Vitamin A. These findings are in agreement with research findings on the deficiency of Vitamin A intake by agriculturalist communities which established that the mothers have a notion that only meat contains Vitamin A nutrients.

Fruit as a source of food rich in Vitamin A was not highly consumed as compared to meat sources. Fish as source of food rich in Vitamin A source was low, accounting for 5.1\%. This low contribution can be attributed to cultural beliefs where the Meru community does not include fish in the diet.
The Meru people traditionally do not consume fish despite of its availability in the major rivers found in the region [20]. The small proportion of fish as source of Vitamin A can be attributed to the availability of fish reared in pond through economic stimulus interventions by the National Government.

On the source of Vitamin A rich foods, it was established that chicken meat and eggs were obtained from the farms while beef meat was bought from butcheries. The study also found that caregivers buy vegetables from market and neighbours $(23.4 \%)$ as compared to $8.1 \%$ who grew in their farms. These can be attributed to the arid nature of the study area which does not support arable farming. These findings are supported by the report on drought situation in Tharaka Nithi County by National Drought Management Authority (NDMA) of 2017 [21]. The report noted that Gatunga ward does not receive adequate rainfall to support crop growing and as a result children suffer from inadequate supply of food nutrients.

The study revealed that only a small number $32(3.4 \%)$ of the respondents bought meats as source of Vitamin A. This can be attributed to low income come levels as noted by this study. It can be argued that the sources of Vitamin A are obtained from the farms and by purchasing. Plant based Vitamin A source foods are mainly cheap and can be readily availed through cultivation in a kitchen garden. This study established that $29.1 \%$ of the caregivers bought vegetables and fruits to provide Vitamin A-rich foods. This observation would mean that the children in the study area were likely to suffer from low Vitamin A intake since they have to purchase such foods. This study established that the household income was low (Table 4) which was not enough to buy specific vitamin rich foods and other household items. These findings are supported by the study by Lorch on causes of VAD found 
that the lack growing of garden green leafy vegetables and fruits has a profound effect on the intake of adequate Vitamin A [22].

On food diversity the study established that the children consumed 15 different foods rich in Vitamin A. The frequency of consumption of food diversity had mixed outcomes with tomatoes $(83.0 \%)$, vegetable oil $(64.0 \%)$ and green leafy vegetables $(58.5 \%)$ being the most consumed food. When the foods were grouped as per FANTA (2009) [19] Vitamin A-rich food groups, Vitamin A-rich plant based foods groups were mostly consumed at 58.5\% followed by eggs $(52.1 \%)$ while fruits based food groups were the least consumed at $7.2 \%$. The child dietary diversity score established that only $41.8 \%$ of the children consumed more than five groups of Vitamin A-rich foods. These revelations mean that majority $(58.2 \%)$ consumed less than four groups of foods rich in Vitamin A. These findings imply that the children in the study area were fed with limited number of Vitamin A-rich foods. It can be argued from the above findings that children aged between 12-59 months studied did not have adequate intake of Vitamin A.

\section{Conclusion}

It also concluded that health workers as the major source of information regarding Vitamin A as compared to other sources. On the benefits of Vitamin A, the study concludes that the caregivers have low understanding of its value. On purchase of food rich in Vitamin A, the study concludes that the caregivers had little capacity based on the little amount spent on such foods. Finally, the study also concludes that the information available on Vitamin A among the caregivers was not adequate to help them make decision on issues of Vitamin A intake. On Vitamin A-rich food diversity, the study concludes that the children were fed with less dietary food diversity with green leafy ones being mostly consumed. Animal based foods were less consumed. Lack of adequate income and lack of reliable rainfall to support growing of food rich in Vitamin A like fruits and vegetables contributed little dietary diversity. Overall based on the above conclusions the children aged 12-59 months does not adequately get Vitamin A from the foods they consume.

\section{References}

[1] Khillan, J. S. (2014). Vitamin A/Retinol and Maintenance of Pluripotency of Stem Cells. Nutrients, 6, 1209-1222.

[2] UNICEF. (2012). Improving child nutrition: The achievable imperative for global progress. New York, USA: United Nations Children's Fund (UNICEF).

[3] Smith, R. G. (2012). The Vitamin Cure for Eye Disease: How to Prevent and Treat Eye Disease Using Nutrition and Vitamin Supplementation. Laguna Beach, USA: Basic Health Publications.
[4] WHO. (2015). Food Safety: What you should know. Retrieved May 20, 2017, from www.searo.who.int: http://www.searo.who.int/entity/world_health_day/2015/whdwhat-you-should-know/en/.

[5] Tidy, C. (2015). Vitamin A Deficiency. Retrieved January 14, 2016, from www.patient.info: https://patient.info/doctor/vitamin-a-deficiency.

[6] UNICEF. (2015). Micronutrients. Retrieved February 22, 2016, from www.unicef.org: https://www.unicef.org/nutrition/index_iodine.html.

[7] UNICEF. (2007). The state of the world's children 2008: Child Survival. New York: UNICEF.

[8] Akhtar, S., Ahmed, A., Randhawa, M. A., Atukorala, S., Arlappa, N., Ismail, T., et al. (2013). Prevalence of Vitamin A Deficiency in South Asia: Causes, Outcomes, and Possible Remedies. Journal of Health Population and Nutrition, 31 (4), 413-423.

[9] Motee, A., \& Jeewon, R. (2014). Importance of Exclusive Breast Feeding and Complementary Feeding Among Infants. Current Research in Nutrition and Food Science: Nutrition Science Journal, 2 (2), 56-72.

[10] Hazir, T., Begum, K., Arifeen, S., Khan, A. M., Huque, H. M., Kazmi, N., et al. (2011). Measuring coverage in MNCH: a prospective validation study in Pakistan and Bangladesh on measuring correct treatment of childhood pneumonia. Journal. pmed, $10(5), 1-12$.

[11] Chiu, M., \& Watson, S. (2015). Xerophthalmia and vitamin A deficiency in an autistic child with a restricted diet. BMJ Case Rep, 10 (1136), 1-3.

[12] Sardesai, V. (2011). Introduction to Clinical Nutrition (3rd ed). Florida, United States: CRC Pres.

[13] Kupka, R., Nielsen, J., Dhillon, C. N., Blankenship, J., Haskell, M. J., \& Baker, S. K. (2016). Safety and Mortality Benefits of Delivering Vitamin A Supplementation at 6 Months of Age in Sub-Saharan Africa. Food and Nutrition Bulletin, 37 (3), 375-386.

[14] Nair, M. K., Augustine, L. F., \& Konapur, A. (2016). FoodBased Interventions to Modify Diet Quality and Diversity to Address Multiple Micronutrient Deficiency. Frontiers in Public Health, 3 (277), 1-14.

[15] Jemberu, Y., Zegeye, M., Singh, P., \& Abebe, H. (2017). Consumption of Vitamin A Rich Foods and Associated Factors among Children Aged 6-23 Months in Kachabira District, Southern Ethiopia. International Journal of Food Science and Nutrition Engineering, 7 (1), 1-10.

[16] Bailey, R. L. (2015). The Epidemiology of Global Micronutrient Deficiencies. Annals of Nutrition and Metabolism, 2015 (2), 22-33.

[17] GoK. (2011). The Kenya National Micronutrient Survey. Nairobi: Government Printers.

[18] KNBS. (2014). Kenya Demographic and Health Survey. Nairobi: Kenya Bureau of Statistics.

[19] FANTA. (2009). Household Dietary Diversity Score (HDDS) for Measurement of Household Food Access Indicator Guide. Washington, DC: Food and Nutrition Technical Assistance (FANTA). 
[20] Barasa, L. (2014, August 13). Meru County government launches fish eating campaign. Retrieved October 12, 2019, from https://www.nation.co.ke: https:/www.nation.co.ke/counties/meru/Meru-fish-eatingcampaign/1183302-2417744-16fr11z/index.html.
[21] NDMA. (2017). Drought Early Warning Bulletin. Nairobi: National Drought Management Authority.

[22] Lorch, A. (2005). Vitamin A deficiency: diverse causes, diverse solutions. London: Greenpeace International. 